\title{
A Case of Intra-abdominal Endocrine Tumor Possibly Arising from an Ectopic Pancreas
}

\author{
Masao Ogata ${ }^{1}$, Naoto Chihara', Tetsuro Matsunobu ${ }^{1}$, Masaki Koizumi ${ }^{1}$, \\ Masanori Yoshino ${ }^{1}$, Takeshi Shioya ${ }^{1}$, Masanori Watanabe ${ }^{1}$, Akira Tokunaga ${ }^{1}$, \\ Takashi Tajiri ${ }^{2}$ and Koshi Matsumoto ${ }^{3}$ \\ ${ }^{1}$ Institute of Gastroenterology, Nippon Medical School Musashi-Kosugi Hospital \\ ${ }^{2}$ Surgery for Organ Function and Biological Regulation, Graduate School of Medicine, Nippon Medical School \\ ${ }^{3}$ Department of Pathology, Nippon Medical School Musashi-Kosugi Hospital
}

\begin{abstract}
We report an intra-abdominal endocrine tumor possibly arising from an ectopic pancreas. A 45-year-old woman visited the Nippon Medical School Musashi-Kosugi Hospital because of right-sided hypochondralgia and upper abdominal discomfort of 1 year's duration. An intraabdominal tumor was diagnosed on the basis of the results of an ultrasound examination, computed tomography and magnetic resonance. Surgery was subsequently performed using laparoscopic techniques, and a tumor without firm adhesions was found near the wall of the duodenal bulbus. The tumor was easily removed; the resected specimen $(55 \times 45 \times 25 \mathrm{~mm}$, $50 \mathrm{~g})$ was composed of bloody fluid within a cystic tumor. Histological and immunohistochemical examinations of the tumor showed a type 3 ectopic pancreas, according to the classification proposed by Heinrich. The patient's recovery was uneventful.
\end{abstract}

(J Nippon Med Sch 2007; 74: 168-172)

Key words: ectopic pancreas, Heinrich classification, endocrine tumor

\section{Introduction}

Symptomatic cases of ectopic pancreas in the wall of the stomach, duodenum, or jejunum have frequently been reported. Reports of ectopic pancreas in other areas, however, are rare. Furthermore, endocrine tumor arising from ectopic pancreas is also rare. Here, we describe a patient with an endocrine tumor that may have arisen from an ectopic pancreas ${ }^{1-4}$.

\section{Case Report}

A 45-year-old woman visited our hospital in 2004 because of right-sided hypochondralgia and upper abdominal discomfort of 1 year's duration. An intraabdominal tumor was diagnosed after detailed examinations had been performed. The patient did not smoke or drink alcohol. No abnormal findings were obtained during a physical examination. Blood tests showed a high serum amylase level (178 IU). Tumor markers, including carcinoembryonic antigen

Correspondence to Masao Ogata, MD, Institute of Gastroenterology, Nippon Medical School Musashi-Kosugi Hospital, 1-396 Kosugi-cho, Nakahara-ku, Kawasaki, Kanagawa 211-8533, Japan

E-mail: masao@nms.ac.jp

Journal Website (http://www.nms.ac.jp/jnms/) 


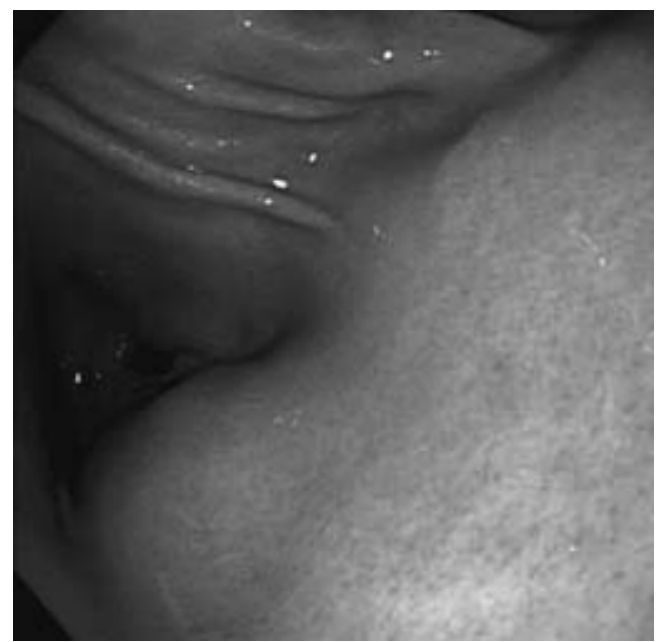

Fig. 1 A gastroscopy reveals a submucosal tumor in the gastric antrum. The mucosa appeares normal throughout the stomach.

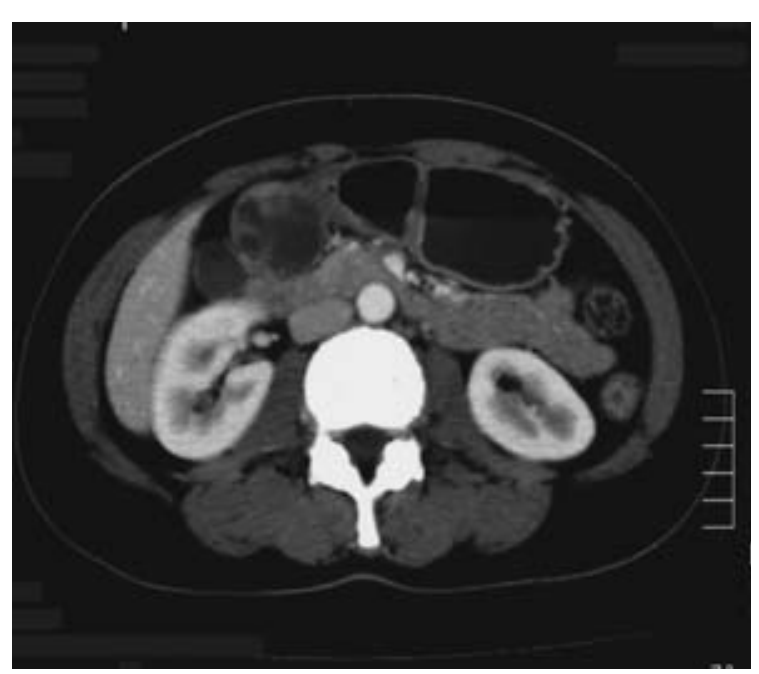

Fig. 2A Abdominal computed tomography reveals an intraabdominal tumor with a spherical cystic component and no enhancement at a position adjacent to the gallbladder and pancreas.

(2.3 ng/ml), CA19-9 $(21 \mathrm{U} / \mathrm{ml})$ and alpha fetoprotein (less than $10 \mathrm{ng} / \mathrm{ml}$ )were within their normal ranges. Gastroscopy revealed a submucosal mass in the gastric antrum; the mucosa was normal (Fig. 1). An abdominal computed tomography (CT) scan revealed an intra-abdominal tumor with a spherical cystic component and no adhesions to the adjacent gallbladder and pancreas head (Fig. 2A). Abdominal ultrasonography demonstrated a tumor $(47 \times 42 \times$ $35 \mathrm{~mm})$ with mixed hyperechoic and hypoechoic components located adjacent to the gallbladder and

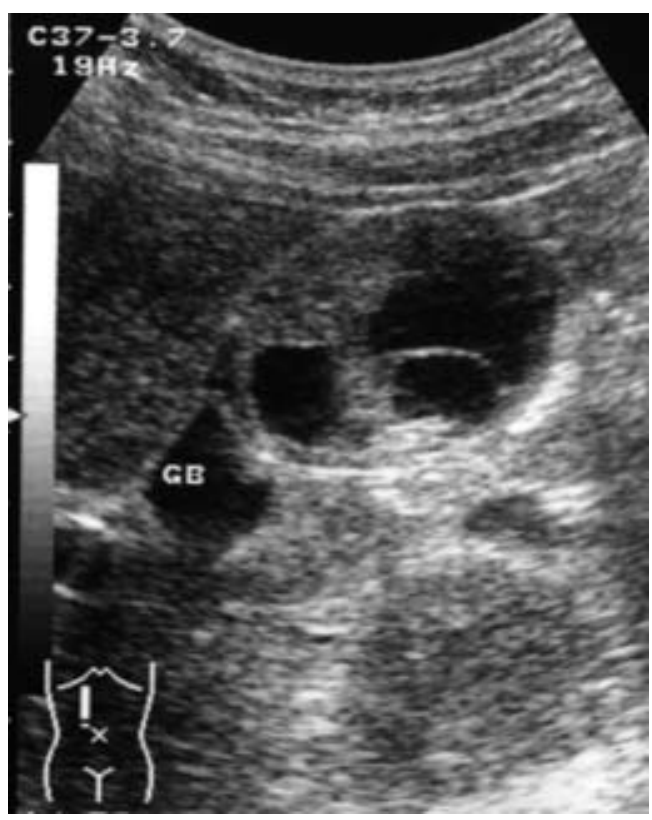

Fig. 2B AUS reveals a tumor $(47 \times 42 \times 35 \mathrm{~mm})$ with mixed hyperechoic and hypoechoic components located adjacent to the gallbladder and the liver.

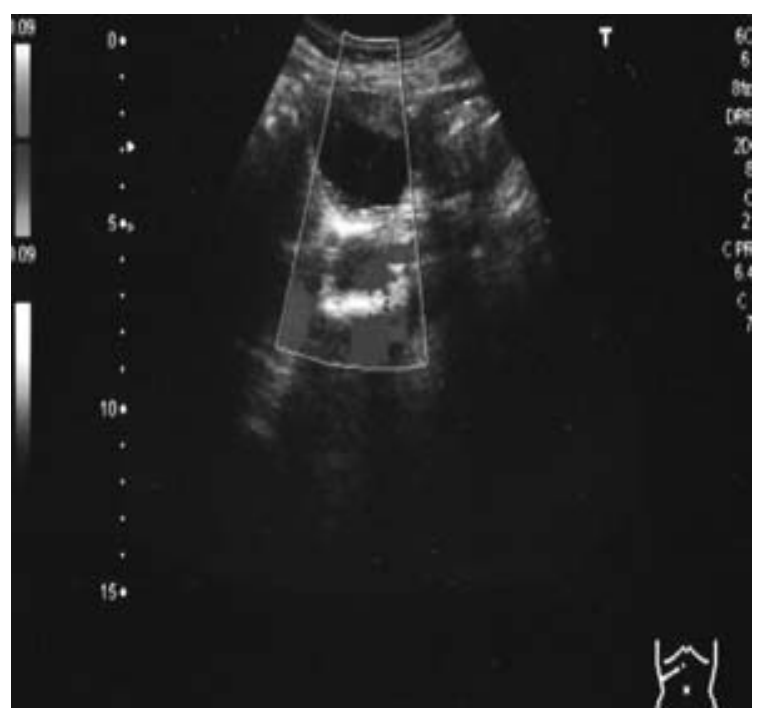

Fig. 2C Color Doppler sonography shows a little signal from the tumor.

the liver (Fig. 2B). Color Doppler sonography showed a little signal from the tumor (Fig. 2C). Magnetic resonance (MR) showed a tumor with a smooth edge and a cystic intensity inside (Fig. 2D). Endoscopic retrograde cholangiopancreatography (ERCP) did not reveal any abnormalities. Abdominal angiography was performed and showed that the arteries feeding the tumor were the posterior superior pancreaticoduodenal artery (PSPDA) and 


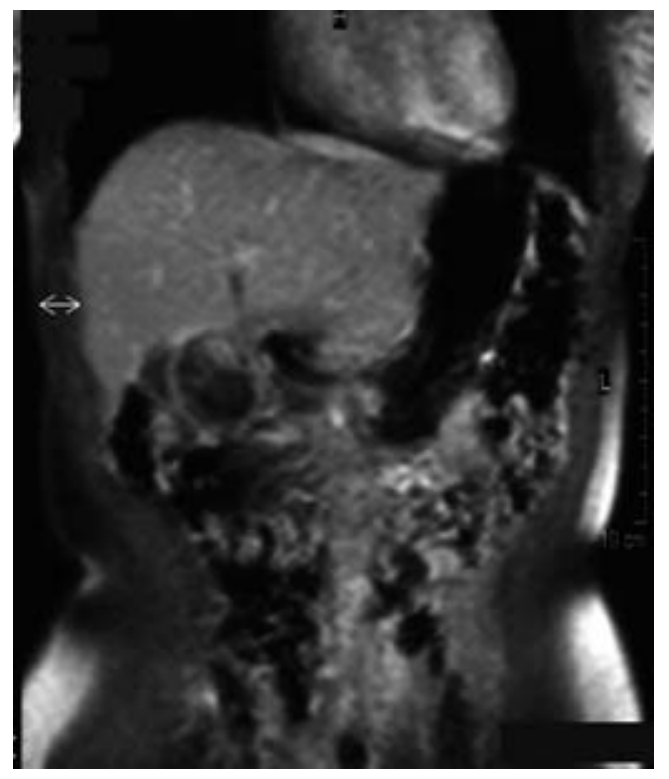

Fig. 2D MR shows a tumor with a smooth edge and a cystic intensity inside. the anterior superior pancreaticoduodenal artery (ASPDA); only the edge of the tumor was enhanced. A laparoscopic tumor resection was performed. The tumor was located near the wall of the duodenal bulbus and was not firmly adherent to the adjacent organs. The tumor was easily removed; the resected specimen was $55 \times 45 \times 25 \mathrm{~mm}$ in size. The tumor contained $70 \mathrm{~m} l$ of sanguineous fluid.

Histopathological studies revealed that the tumor was composed of small to medium-sized epithelial cells with round nuclei, forming solid nests, trabeculae, ribbons, and tubules with occasional vascular pseudorossettes (Fig. 3). Immunohistochemical staining for chromogranin, synaptophysin, keratin, and serotonin was positive, whereas that for grimelius, insulin, and vimentin was negative. Immunohistochemical staining for gastrin, glucagon, somatostatin, and carcinoembryonic antigen was negative (data not shown). The MIB-1 labeling index was less than $5 \%$ (Fig. 4). The histological diagnosis was an endocrine tumor that had possibly arisen from an ectopic pancreas. The
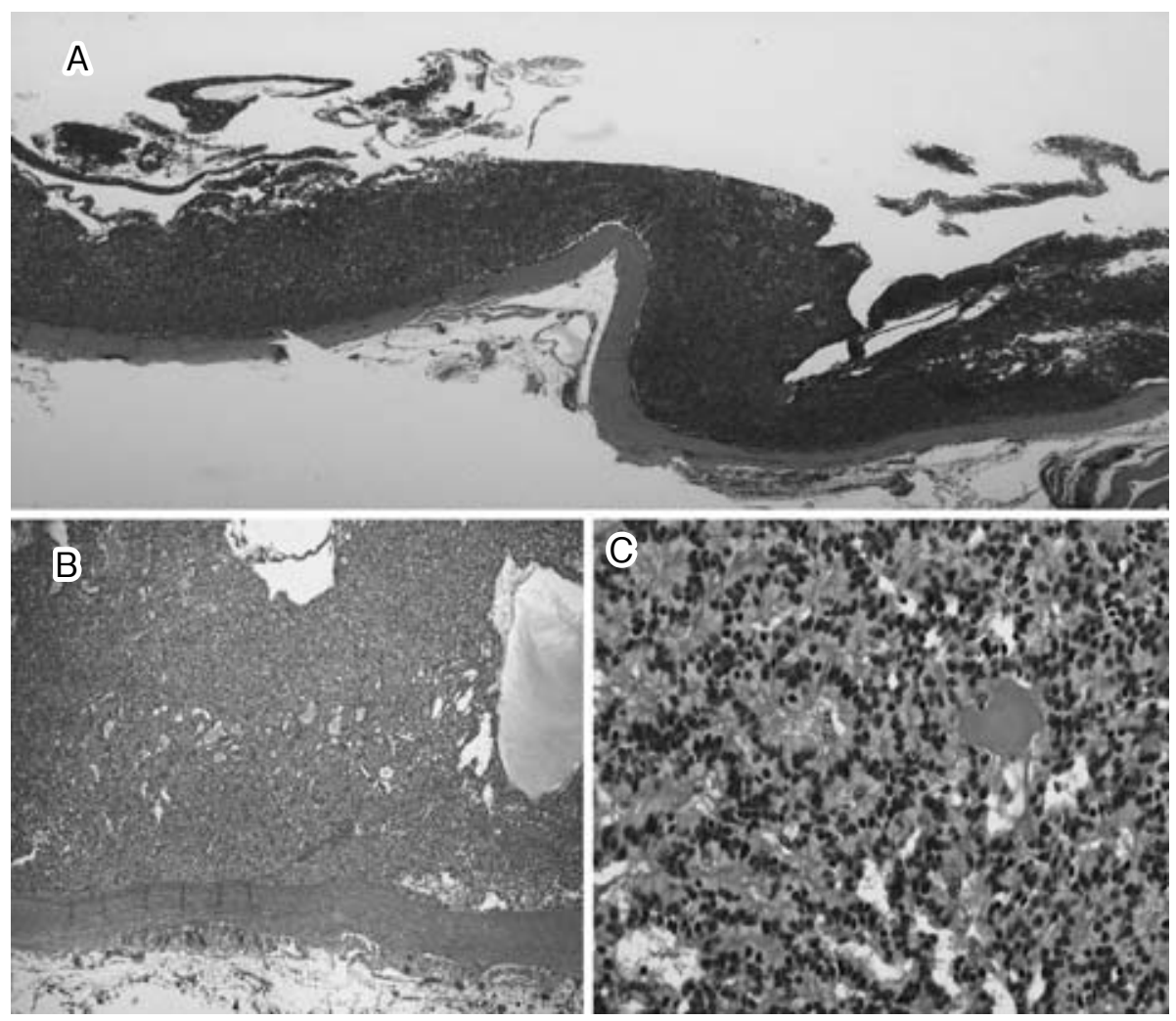

Fig. 3 HE staining shows a rosette, ribbon and glandular pattern of cell growth and round-shaped nuclei. $(\mathrm{A}: \times 5, \mathrm{~B}: \times 10, \mathrm{C}: \times 100)$ 

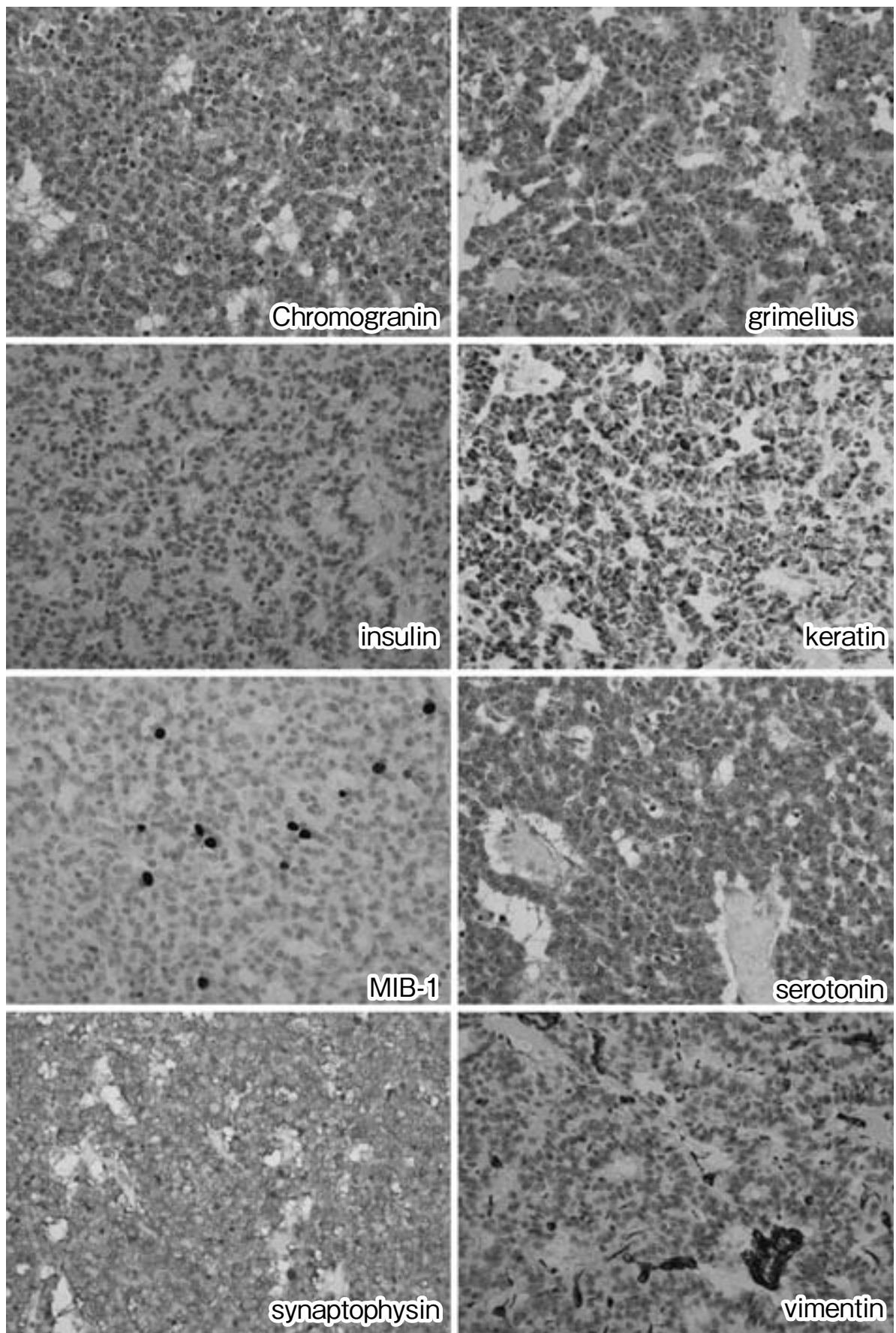

Fig. 4 Immunohistological stainings were positive for chromogranin, synaptophysin, keratin, and serotonin and negative for grimelius, insulin, and vimentin. MIB-1 labeling index was less than $5 \% .(\times 100)$

patient remains well 27 months after surgery.

\section{Discussion}

Ectopic pancreases are usually small, measuring 3 $\mathrm{cm}$ or less in diameter; however, gastric lesions tend to be larger than those located at other sites. The classic radiologic and endoscopic features of ectopic pancreas are a well-circumscribed intramural nodule with a central umbilication that may be the site of ductal drainage to the mucosal surface ${ }^{5}$. Umbilication is observed in less than half of cases; therefore, ectopic pancreases must be differentiated from other intramural masses, such as gastrointestinal stromal 
Table 1 Heinrich classification of Ectopic Pancreas ${ }^{8}$

Type 1 Ectopic tissue with acini, ducts and islets of Langerhans.

Type 2 Ectopic tissue with incomplete or lobular arrangement (only a few acini and multiple ducts). Endocrine elements are absent.

Type 3 Ectopic tissue of proliferating ducts (so-called adenomyoma). Both exocrine acini and endocrine elements are lacking.

tumors and metastatic carcinomas ${ }^{6,7}$.

We report an intra-abdominal tumor that was diagnosed using ultrasound, CT and MR. The tumor was thought to be an endocrine tumor arising from an ectopic pancreas. Diagnosing tumors derived from ectopic pancreases is difficult. In the present case, the tumor was free of adjacent organs, such as the duodenum, gallbladder, and liver. Macroscopically, the cut surface of the tumor was cystic with a hard wall.

Histological examination of the tumor showed that the tumor was composed of small to medium-sized epithelial cells with round nuclei, forming solid nests, trabeculae, ribbons, and tubules with occasional vascular pseudorossettes. Immunohistochemical staining for chromogranin A, synaptophysin, keratin, serotonin was positive. On the basis of these comprehensive histological and immunohistochemical examinations, the lesion was diagnosed as an intra-abdominal endocrine tumor possibly arising from an ectopic pancreas. There was no malignant potential, as indicated by the MIB1 labeling index of, less than 5\%.

Ectopic pancreas has been classified into three types by Heinrich $(\text { Table } \mathbf{1})^{8}$. Some type 3 cases lack acinar tissue entirely. The current case was considered to be a type 3 ectopic pancreas, based on Heinrich's classification. An endocrine tumor arising from an ectopic pancreas is a rare with only one previously documented case to our knowledge $\mathrm{e}^{9-11}$.

\section{References}

1. Jaffer R: The pancreas. In Textbook of fetal and perinatal pathology, Vol. 2 (Wigglesworth JS, Singer DB, ed), 1991; pp 1021-1055, Blackwell Scientific, Boston. Massachusetts.

2. Hickman DM, Frey CF, Carson JW: Adenocarcinoma arising in gastric heterotopic pancreas. West J Med 1981; 135: 57-62.

3. Edward C.S. Lai, Ronald K. Tompkins: Heterotopic Pancreas. Am J Surg 1986; 151: 697-700.

4. Barbosa JJ, Dockerty MB, Waugh JM, et al: Pancreatic heterotopia: Review of the literature and report of 41 authenticated surgical cases of which 25 were clinically significant. Surg Gynecol Obstet 1946; 82: $527-542$.

5. Kilman WJ, Berk RN: The spectrum of radiographic features of aberrant pancreatic rests involving the stomach. Radiology 1977; 123: 291-296.

6. Lyska E, Lester J: Adenocarcinoma arising in association with gastric heterotopic pancreas. J Surgical Oncology 2004; 87: 53-57.

7. Nakao T, Yanoh K, Itoh A: Aberrant pancreas in Japan. Reviw of the literature and report of 12 surgical cases. Med J Osaka University 1980; 30: 5763.

8. von Heinrich H: Ein Beitrag zur Histologie des sogen: Akzessorischen Pankreas. Virchows Arch A Pathol Anat Histopathol 1909; 198: 392-401.

9. Tolentino LF, Lee H, Maung T, Stabile BE, Li K, French SW: Islet cell tumor arising from a heterotopic pancreas in the duodenal wall with ulceration. Exp Mol Pathol 2004; 76: 51-56.

10. Hammock L, Jorda M: Gastric endocrine pancreatic heterotopia. Arch Pathology Lab Med 2002; 126: 464467.

11. Fukunaga $\mathrm{M}$ : Gastric carcinoma resembling pancreatic mixed acinar-endocrine carcinoma. Hum Pathol 2002; 33: 569-573.

(Received, January 9, 2007)

(Accepted, January 30, 2007) 